\title{
Rectal biopsy and precancer in ulcerative colitis
}

\author{
H. E. MYRVOLD, N. G. KOCK, AND CHR. ÅHREN \\ From the Departments of Surgery III and Pathology II, Sahlgrenska Sjukhuset, University of Göteborg, \\ Göteborg, Sweden
}

SUMMARY Forty-seven patients with ulcerative colitis subjected to elective proctocolectomy were investigated with regard to the occurrence of precancerous changes in the rectal biopsy and the operation specimen. In the rectal biopsy from seven patients precancerous lesions were found and in the operation specimens from these patients precancer was also found in other parts of the colon. In five of these patients the precancer was associated with carcinoma and in four of the patients the tumour had not been detected before operation. It is concluded that rectal biopsy is of great value in patients with ulcerative colitis for detection of early cancer and patients at risk of developing a colitis carcinoma.

It is well documented that patients with ulcerative colitis have an increased incidence of carcinoma in the colon compared with the normal population. The most important predisposing factors are the duration of the disease and the extent of involvement of the colon. In patients with total colitis the cumulative expected incidence of carcinoma will amount to approximately $40 \%$ after 25 years (Goligher, 1970; Devroede, Taylor, Sauer, Jackman, and Stickler, 1971). However, in any one patient the risk of developing carcinoma is unpredictable. Furthermore, the diagnosis of an early carcinoma is often missed by the usual diagnostic tools and the tumour incidentally found at laparotomy or in the removed specimen after colectomy for ulcerative colitis.

The occurrence of precancerous changes in the colonic mucosa of patients with ulcerative colitis associated with carcinoma was described as long ago as in 1949 by Warren and Sommers and in 1959 by Dawson and Pryse-Davies. In 1967 Morson and Pang described precancerous changes in the colonic and rectal mucosa of 23 colectomy specimens removed for colitis carcinoma. These precancerous changes were extensive and involved areas of the mucosa distant from sites of carcinoma as well as in their vicinity. In 16 out of 148 retrospectively studied rectal biopsies precancerous changes were found and in the colectomy specimens similar changes were seen but without invasive carcinoma. In a prospective study the same authors found precancerous lesions in rectal biopsies from nine patients

Received for publication 5 November 1973. with total colitis and at operation carcinoma was present in five of them.

Morson and Pang emphasized that rectal biopsy was an aid to cancer control in ulcerative colitis and they recommended that proctocolectomy should be carried out when precancerous changes were found in a rectal biopsy.

Hultén, Kewenter, and Åhrén (1972), in a retrospective study, confirmed the occurrence of precancerous changes in ulcerative colitis associated with carcinoma and claimed that the occurrence of precancerous changes in a rectal biopsy justifies prophylactic colectomy.

Evans and Pollock (1972), on the other hand, reported that the extent of precancer was very variable in four cases with colitis carcinoma and in all cases the rectum was spared wholly or in part. They claimed that rectal biopsy will fail to indicate the development of malignancy in some patients with ulcerative colitis.

The aim of the present prospective study was to evaluate the validity of rectal biopsy for detecting precancerous lesions and possible early carcinomas in patients with ulcerative colitis.

\section{Material and Methods}

The material comprised 47 patients, 29 females and 18 males, with ulcerative colitis involving the whole of the large bowel. They had all been referred to our department for elective proctocolectomy and construction of a continent ileostomy (Kock, 1973).

Before the operation all patients had barium 
enema studies and three to four rectal biopsies were obtained and examined histologically for the occurrence of reactive cellular atypia and precancerous changes. All biopsies and $x$-ray investigations referred to were carried out less than six months before proctocolectomy. The preoperative findings were correlated to the occurrence of precancer and carcinoma in the colectomy specimens. The operative specimens were examined systematically including total cross sections of the rectum at the 5,10 , and $20 \mathrm{~cm}$ level.

The criteria for the diagnosis of precancer were the changes described by Morson and Pang (1967), including loss of nuclear polarity as stressed by Hultén et al (1972). The histological examination of rectal biopsies and operative specimens was done by one and the same pathologist ( hrén). The present study is based on the findings in the original routine histological examination and no retrospective re-examination has been made.

\section{Results}

In 24 patients no precancer or atypia was found in any of the rectal biopsies but when the operative specimens were examined cellular atypia was found in nine and precancerous changes in one of the specimens (table I). Cellular atypia but not the 'full picture' of precancerous changes was found in the rectal biopsies from 16 patients. Examination of the operative specimens disclosed precancer in 10 of them. Precancerous changes were found in rectal biopsies from seven patients (fig 1). Examination of the operative specimens revealed precancer in all of them, and also carcinoma in five. In only one of the patients with carcinoma had the tumour diagnosis been established before the operation. In the operative specimens the whole mucous membrane showed precancerous changes in two cases, while the rest disclosed patchy distribution of the lesion. In two cases no precancer was found in the rectum of the operation specimens, although precancer occurred at other sites in the colon.

\begin{tabular}{lllll}
\hline Rectal Biopsy & \multicolumn{3}{l}{ Operative Specimen } \\
\cline { 2 - 4 } & $\begin{array}{l}\text { No Atypia or } \\
\text { Precancer }\end{array}$ & $\begin{array}{l}\text { Cellular } \\
\text { Atypia }\end{array}$ & $\begin{array}{l}\text { Precancer } \\
\text { (Carcinoma) }\end{array}$ \\
\hline $\begin{array}{l}\text { No atypia or } \\
\text { precancer }\end{array}$ & $(24)$ & 14 & 9 & 1 \\
$\begin{array}{c}\text { Cellular } \\
\text { atypia }\end{array}$ & (16) & 1 & 5 & 10 \\
$\begin{array}{l}\text { Precancer } \\
\text { (7) }\end{array}$ & 0 & 0 & $7(5)$ \\
\hline
\end{tabular}

Table I Occurrence of cellular atypia and precancer in rectal biopsies and in operation specimens of 47 patients with ulcerative colitis

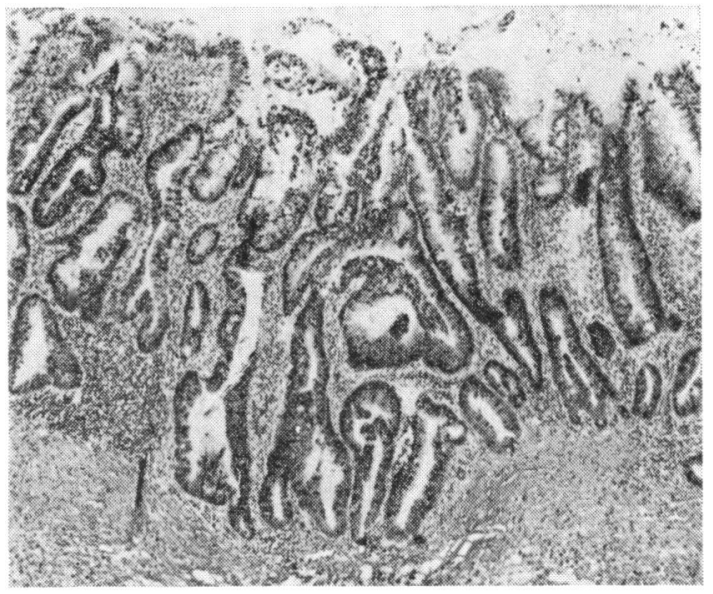

Fig. 1A

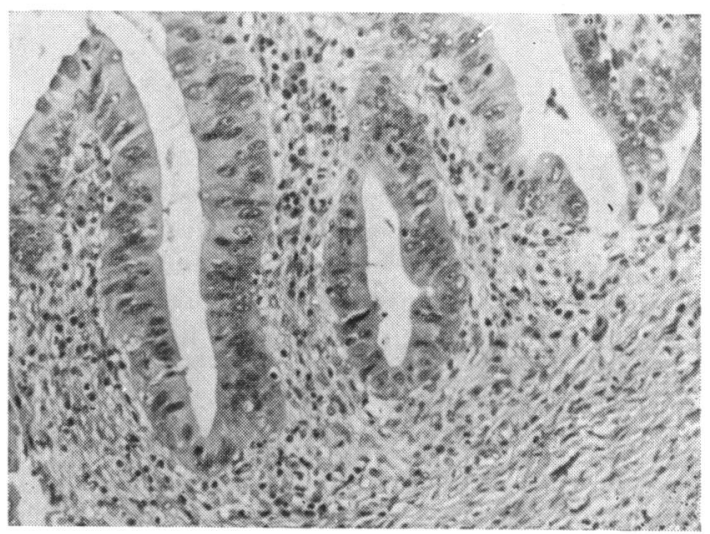

Fig. 1B

Fig $1 A \times 60$ et $B \times 190 v$. Gieson. Preoperative rectal biopsy showing precancerous lesion with lateral budding, hyperchromatic and stratified nuclei $(A)$ and in higher magnification loss also of nuclear polarity $(B)$.

Precancerous lesions were found in the operation specimens of 14 out of 33 patients $(42 \%)$ under the age of 40 years and in four out of 14 patients $(28 \%)$ above 40 years of age (fig 2). Two patients with colonic carcinoma were only 18 and 27 years old respectively.

Precancer was present in 11 out of $35(32 \%)$ operation specimens from patients with a history of less than 10 years and in seven out of 12 specimens from patients with a history exceeding 10 years $(58 \%)$. Carcinoma was present in two out of 35 patients $(6 \%)$ with a duration of less than 10 years and in three out of 12 patients $(25 \%)$ with a duration of more than 10 years (fig 3 ). 


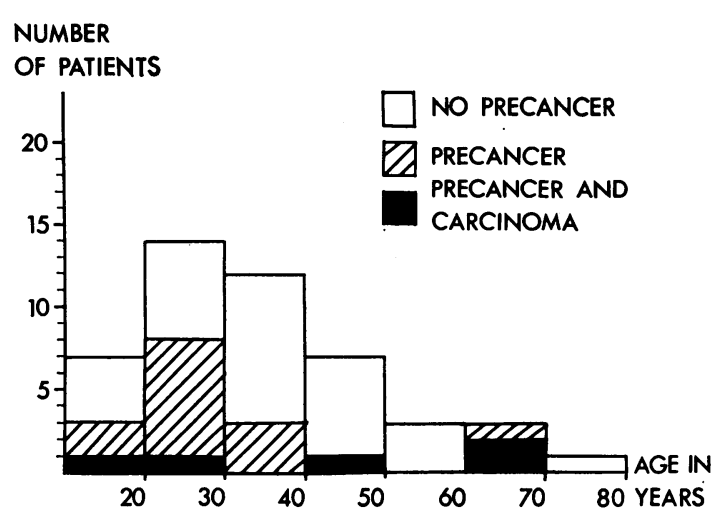

Fig 2 Patients grouped with respect to age at operation. The occurrence in the operation specimens of carcinoma (+precancer) and precancer only indicated in the bars.

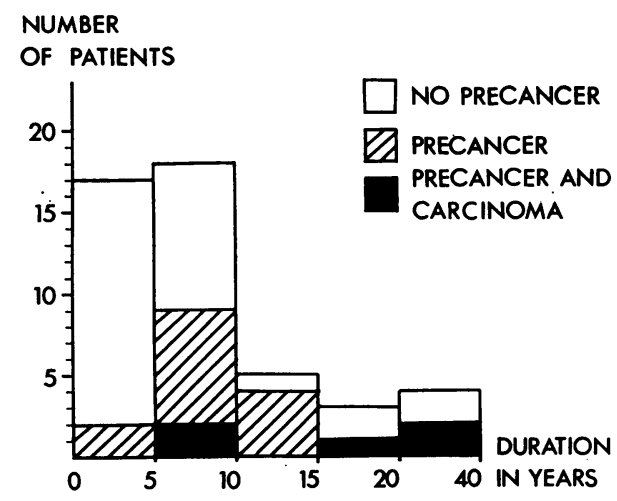

Fig 3 Patients grouped with respect to the duration of colitis symptoms. The occurrence in the operation specimens of carcinoma (+precancer) and precancer only indicated in the bars.

\section{CASE SUMMARIES OF THE FIVE}

PATIENTS WITH CARCINOMA

\section{Case 1}

Aged 27 years, female. Duration 10 years. Barium enema: total colitis, no tumour. Rectal biopsy, precancer: proctocolectomy. Pathological diagnosis: total colitis with early invasive carcinoma in transverse colon. Precancer in the whole colon and rectum. The patient is well more than one year after the operation.

\section{Case 2}

Aged 68 years, female. Duration 25 years. After five years of colitis a caecostomy had been performed during an acute attack of colitis. Twenty years later she was admitted for operation. Barium enema, total colitis, no tumour. Rectoscopy, colitis, no clear-cut tumour. Biopsy, precancer, proctocolectomy. Pathological diagnosis: precancerous changes throughout the colon and rectum and in the rectum a small adeno-carcinoma of medium grade of malignancy. The patient died postoperatively.

\section{Case 3}

Aged 18 years, male. Duration six years. Barium enema: total colitis, no tumour. Rectal biopsy, precancer: proctocolectomy. Pathological diagnosis, precancerous changes throughout the colon and rectum and in sigmoid an early adenocarcinoma of medium degree of malignancy (size $2 \times 3 \mathrm{~cm}$ ). The patient is well six months after the operation.

\section{Case 4}

Aged 44 years, female. Duration 23 years. Barium enema: total colitis, no tumour. Rectal biopsy, precancer: proctocolectomy. Pathological diagnosis, total colitis with precancerous changes throughout the colon. No tumour was found. Four years later a perineal tumour was removed. Microscopical examination: metastasis from a colonic colloid adenocarcinoma. She died from pulmonary embolism after pelvic evisceration for local recurrence involving the pelvic organs six years after the first operation.

\section{Case 5}

Aged 67 years, female. Duration 40 years. Hemicolectomy performed for carcinoma in the ascending colon three years before she was admitted to our department. Rectal biopsy, precancer: proctocolectomy. Pathological diagnosis, total colitis and precancerous changes in the remaining colon and rectum.

The patient died with pulmonary metastasis three years after the last operation.

\section{Discussion}

The primary aim of the present investigation was to evaluate in patients with ulcerative colitis the accuracy of routine histological examination of rectal biopsies for detection of patients at risk of developing carcinoma or for detection of established carcinomas. During the examination of the rectal biopsies extreme care was taken by the pathologist to avoid false-positive diagnoses and in the present study no such false statement was found. This caution also explains in part the high number of rectal biopsies classified as cellular atypia although 
precancerous changes were found in the colectomy specimens.

As pointed out earlier (Hultén et al, 1972), there are in some cases considerable difficulties in differentiating between reactive atypia caused by severe inflammation and true precancerous changes. The pathologist might overcome this difficulty to a certain extent by increasing his personal experience of this special diagnostic problem. Nevertheless, there will always remain a certain number of biopsies in which it is impossible to differentiate between simple reactive atypia and true precancer. In 10 out of 16 cases classified as cellular atypia from the rectal biopsies, examination of the operation specimens disclosed precancer. The difficult differentiation between inflammatory cellular atypia and precancer and the fact that precancer is in most cases patchy and in some cases does not involve the rectum at all (Evans and Pollock, 1972) means, however, that in a certain number of colitis patients with precancer it has to be expected that the rectal biopsies do not reveal these changes. In this study three to four biopsies were taken from different sites but precancer was usually noted in only one or two of these. In spite of the above facts limiting the usefulness of rectal biopsy, it can be stated on the basis of the present findings that histological examination of rectal biopsies is definitely of great value in selecting patients with ulcerative colitis for operation. Thus, in all patients with precancer in the rectal biopsy, precancer or precancer associated with carcinoma was also found in the operation specimens. Moreover, in only one of the five patients with carcinoma had the tumour been diagnosed preoperatively despite the fact that barium enemas and sigmoidoscopies had been carried out less than six months before operation.

The fact that rectal biopsy disclosed precancer in 11 patients with a duration of the disease of less than 10 years and that two of these patients had already developed a small carcinoma is of considerable importance when discussing the risk of carcinoma in ulcerative colitis. Moreover, precancerous lesions were also found in two patients with a history of less than five years. Although the exact timing of the beginning of the disease may be difficult, the present study calls in question the generally accepted view that patients with ulcerative colitis are seldom at risk of developing carcinoma when the duration of the disease is less than 10 years. The $6 \%$ incidence of carcinoma in the present series of patients with a history of ulcerative colitis for less than 10 years has to be considered when evaluating the indications for operation in patients with a short history of the disease. Furthermore, nine patients with a short history had precancerous lesions, although without developed carcinoma, and have therefore to be regarded as having been at risk of developing carcinoma. The incidence of carcinoma and precancer found in the present investigation does not of course reflect the true incidence in an unselected series of patients with ulcerative colitis but nevertheless shows that the risk of developing cancer, even with a short history of the disease, cannot be neglected.

At the present time there is neither definite proof that carcinoma will develop in all patients with precancerous lesions nor do we know when this will occur. Many facts, however, indicate that in some patients with ulcerative colitis the entire inflamed large bowel mucosa is in a premalignant state and prone to develop carcinoma (Morson and Pang, 1967; Hultén, Kewenter, and Kock, 1971; Hultén et al, 1972). With the present state of knowledge precancer in a rectal biopsy has therefore to be considered as indicating a premalignant condition of the large bowel mucosa and justifying prophylactic proctocolectomy. This is also true when such lesions are found in patients with short histories and in patients with a mild course of the disease.

\section{References}

Dawson, I. M. P., and Pryse Davies, J. (1959), The development of carcinoma of the large intestine in ulcerative colitis. Brit. $J$. Surg., 47, 113-128.

Devroede, G. J., Taylor, W. F., Sauer, W. G., Jackman, R. J., and Stickler, G. B. (1971). Cancer risk and life expectancy of children with ulcerative colitis. New Engl. J. Med., 285, 17-21.

Evans, D. J., and Pollock, D. J. (1972). In-situ and invasive carcinoma of the colon in patients with ulcerative colitis. Gut, 13, 566-570.

Goligher, J. C. (1970). Surgery of the Anus, Rectum, and Colon, 2nd ed. Baillière, Tindall and Cassell, London.

Hultén, L., Kewenter, J., and Ahrén, C. (1972). Precancer and carcinoma in chronic ulcerative colitis. Scand. J. Gastroent., 7, 663-669.

Hultén, L., Kewenter, J., and Kock, N. G. (1971). The long term results of partial resection of the large bowel for intestinal carcinomas complicating ulcerative colitis. Scand. J. Gastroent., 6, 601-604.

Kock, N. G. (1973). Continent ileostomy. Prog. Surg., 12, 180-201.

Morson, B. C., and Pang, L. S. C. (1967). Rectal biopsy as an aid to cancer control in ulcerative colitis. Gut, 8, 423-434.

Warren, S., and Sommers, S. C. (1949). Pathogenesis of ulcerative colitis. Amer. J. Path., 25, 657-679. 